\title{
Transformation dislocations and transformation induced crystal defects in CoFe alloys
}

\author{
T. Waitz and H.P. Karnthaler \\ Institute of Materials Physics, University of Vienna, Boltzmanngasse 5, 1090 Vienna, Austria
}

\begin{abstract}
In CoFe alloys the transformation dislocations and the transformation induced defects were analysed by both conventional transmission electron microscopy and high-resolution transmission electron microscopy methods. The thermally induced transformation $\mathrm{fcc} \rightarrow \mathrm{dhcp}$ occurs by the glide of transformation dislocations that all have the same Shockley partial Burgers vector and therefore cause large shear strains. Plastic deformation near the front of the glissile interface activates glide dislocations in the fcc parent phase. Their subsequent reactions with the transformation dislocations lead to a high density of grown-in dislocations and stacking faults in the dhcp lattice.
\end{abstract}

\section{INTRODUCTION}

Martensitic phase transformations are frequently connected with transformation strains as in the case of shape memory alloys. In Co alloys the transformation occurs between different close packed lattices. The required change of the stacking sequence can be achieved by the movement of transformation dislocations [1]. The transformation dislocations have a Shockley partial Burgers vector (p) and can have also a small component perpendicular to their close packed glide plane. The transformation dislocations can form a glissile interface (transformation front).

In $\mathrm{CoFe}$ alloys a martensitic transformation occurs between the face centred cubic (fcc) high temperature phase and the double hexagonal close packed (dhcp) phase [2]. The transformation temperature decreases with increasing $\mathrm{Fe}$ content from $\sim 400^{\circ} \mathrm{C}$ to room temperature (at about $6 \mathrm{at} \% \mathrm{Fe}$ ) showing a large hysteresis on heating and cooling. The change of the stacking sequence of the close packed layers from $A B C$ ( $\mathrm{fcc}$ ) to $\mathrm{ABAC}$ (dhcp) can be achieved by a pair of transformation dislocations (on adjacent close packed planes) gliding on every fourth close packed plane [3]. It is the purpose of the present study to analyse the transformation dislocations at the transformation fronts by transmission electron microscopy (TEM). In addition, the transformation induced dislocations and stacking faults are analysed by both TEM and high-resolution transmission electron microscopy (HRTEM) methods.

\section{EXPERIMENTAL PROCEDURE}

Rod shaped CoFe alloys of the nominal compositions Co- 1.5 and 6.0 at.\%Fe were made of high purity components (4N8, Johnson \& Matthey). From Co- 1.5 at.\%Fe a single crystal of the fcc phase was grown by a modified Bridgman method in a 'soft' mould of sintered $\mathrm{Al}_{2} \mathrm{O}_{3}$ powder under vacuum $\left(<10^{-3} \mathrm{~Pa}\right)$ at a speed of $15 \mathrm{~mm} / \mathrm{h}$. Immediately afterwards the crystal was cyclically annealed in the fcc phase for about $24 \mathrm{~h}$ at a temperature of $-1350^{\circ} \mathrm{C}$ to reduce the density of grown-in dislocations. The temperature was finally lowered to $500^{\circ} \mathrm{C}$ and the fcc single crystal was moved out of the hot zone at a speed of about $50 \mathrm{~mm} / \mathrm{h}$ thereby achieving the transformation $\mathrm{fcc} \rightarrow \mathrm{dhcp}$ during cooling to room temperature (RT). As confirmed by X-ray methods the crystal contained four dhcp variants corresponding to the four $\{111\}_{\mathrm{fcc}}$ habit planes. The maximum length of a single variant was about $15 \mathrm{~mm}$. Contrary to the Co- $1.5 \mathrm{at} . \% \mathrm{Fe}$ alloy $\mathrm{Co}-6.0$ at. \%Fe was still in the high temperature phase (fcc) at RT.

For the TEM investigations discs with a $[11 \overline{2} 0]_{\mathrm{dhcp}}=[1 \overline{1} 0]_{\mathrm{fcc}}$ foil normal $(\mathbf{F N})$ were prepared by spark erosion. Tiny discs had to be used since CoFe alloys are ferromagnetic. TEM foils were obtained by 
jet polishing and by a final bench polishing both done at RT. The specimens were examined at RT in a Philips CM30 ST operating at 300kV. In addition, the fcc Co- 6.0 at.\%Fe TEM foils were transformed to dhcp by in-situ cooling in the electron microscope.

Light optical investigations were carried out on smooth, electropolished surfaces (surface normal $[11 \overline{2} 0]_{\mathrm{dhcp}}=[1 \overline{1} 0]_{\mathrm{fcc}}$. Specimens of Co- 6.0 and $1.5 \mathrm{at} \% \mathrm{Fe}$ were cooled to about $-170^{\circ} \mathrm{C}$ and heated up to about $480^{\circ} \mathrm{C}$ under vacuum $\left(<10^{-4} \mathrm{~Pa}\right)$, respectively. Afterwards the specimens were examined at $\mathrm{RT}$ in detail in the light optical microscope using differential interference contrast (DIC) and two beam interference methods.

\section{EXPERIMENTAL RESULTS}

\subsection{The fcc $\rightarrow$ dhcp transformation}

\subsubsection{TEM in-situ investigations}

During in-situ cooling in the TEM dhcp lamellae appeared at about $-60^{\circ} \mathrm{C}$ in the fcc parent phase of Co6.0at.\%Fe. The lamellae (lying on the close packed habit planes $\{111\}_{\mathrm{fcc}}$ ) emerged from the bulk regions of the specimens and moved towards the thin edge of the TEM foil. It is interesting to note that the first dhcp lamellae were observed when the stacking fault energy was still positive $\left(9 \pm 2 \mathrm{~mJ} / \mathrm{m}^{2}\right.$, as measured by the dissociation width of fcc glide dislocations). The transformation caused long-range lattice strains leading to the bending of the TEM foils and to the formation of cracks near the edge.

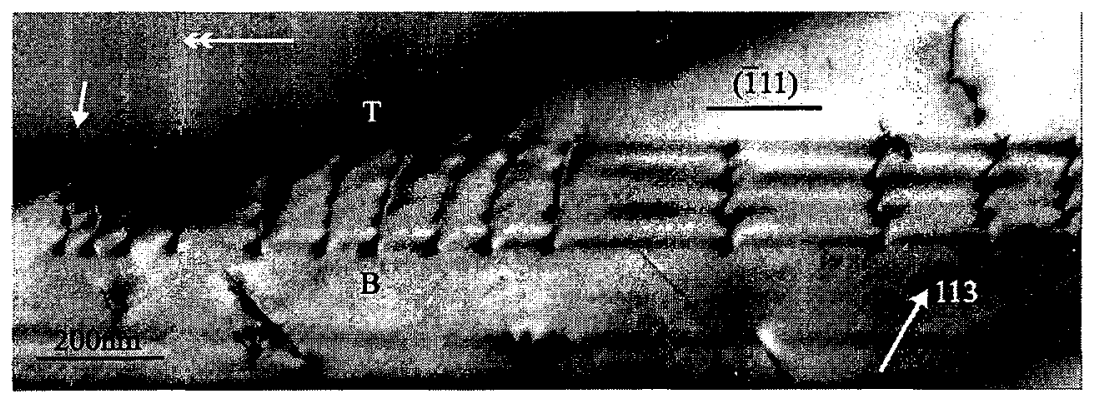

a)

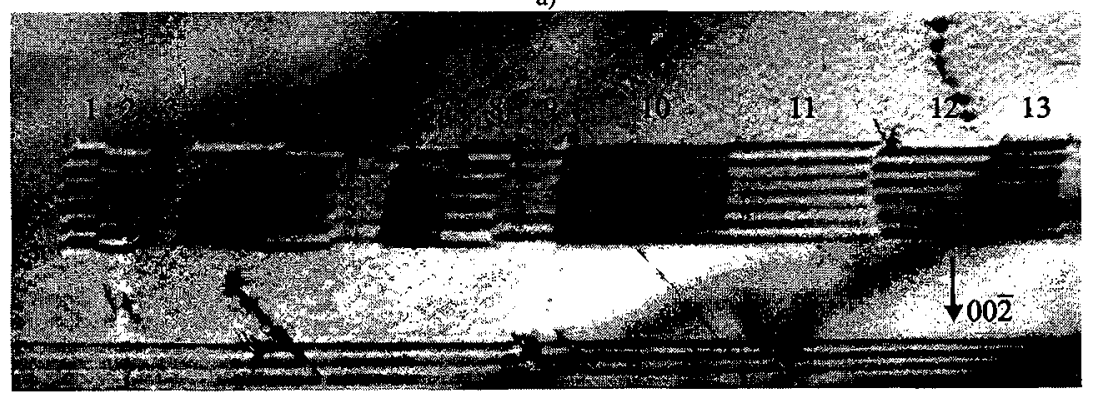

b)

Figure 1: $\mathrm{Co}-6.0 \mathrm{at} \% \mathrm{Fe}$; fcc phase. $\left(\mathbf{B D} \approx \mathbf{F N}=[1 \overrightarrow{10}]_{\mathrm{fcc}}\right)$. TEM bright field images of a dhcp transformation front. The front is emerging during the in-situ transformation $\mathrm{fcc} \rightarrow \mathrm{dhcp}$ from the bulk regions towards the thin areas of the TEM foil (the double arrow indicates the direction of the movement). (a) The transformation dislocations are showing strong contrast $\left(\mathrm{g}=[113]_{\mathrm{fcc}}=[11 \overrightarrow{24}]_{\mathrm{fcc}}\right)$. All dislocations have the same Shockley partial Burgers vector $\left(\mathbf{p}=1 / 6[211]_{\mathrm{fcc}}\right)$ and are in a pile-up configuration. The leading dislocation is marked by an arrow. (b) The lapering dhcp lamella (running along the ( $\overline{1} 11) \mathrm{fcc}$ habit plane) shows contrast modulations similar to overlapping stacking faults (the numbers indicate the thickness in units of $0.8 \mathrm{~nm}$; $\mathbf{g}=[00 \overline{2}]_{\mathrm{fcc}}$ ). At each transformation dislocation the thickness of the lamella increases by $0.8 \mathrm{~nm}$ (i.e. 4 close packed layers). Note the reduced contrast at 3,6 and 9 overlapping faults. 
Fig. 1 shows the glissile transformation front of a dhcp lamella. In the bright field image of Fig. 1a $\left(\mathrm{g}=[113]_{\mathrm{fcc}}=[11 \overline{24}]_{\mathrm{dhcp}}\right)$ the transformation dislocations show strong contrast whereas the lamella shows weak residual contrast only (the leading transformation dislocation is marked by an arrow and the direction of the movement of the lamella is indicated by a double arrow). The contrast of the transformation dislocations was analysed by TEM bright field methods applying different $g$ vectors and using image simulation. As a result it is found that all transformation dislocations have the same Shockley partial Burgers vector $p=1 / 6[211]_{\mathrm{fcc}}$; each dislocation is bounding an extrinsic fcc stacking fault [4]. The lamella is tapering since at each transformation dislocation the thickness of the lamella decreases by $0.8 \mathrm{~nm}$ (i.e. four close packed layers). In the TEM projection the lamella appears as a sequence of overlapping stacking faults (cf. Fig. 1 b). In $\mathbf{g}=[00 \overline{2}]_{\mathrm{fcc}}$ the transformation dislocations are out of contrast whereas the dhcp lamella shows strong contrast oscillations (note the reduced contrast at a thickness of 3 , 6 and 9 overlapping stacking faults [5]). At the transformation fronts of other lamellae formed during insitu heating up to about 350 transformation dislocations were observed in a pile-up configuration all of them having the same Burgers vector.

\subsubsection{Surface investigations}

At polished crystal surfaces striations were observed after the transformation $\mathrm{fcc} \rightarrow \mathrm{dhcp}$ by light optical methods in Co- 6.0 at.\%Fe (cf. Fig. 2a). The striations were found parallel to the traces of all four $\{111\}_{\text {fcc }}$ habit planes of the fcc parent phase and correspond to regions of local tilt as measured by two beam light interference microscopy. Similar, in $\mathrm{Co}-1.5 \mathrm{at} . \% \mathrm{Fe}$ fine striations were observed running along the $(0001) /(111)$ habit plane after a full transformation cycle dhcp $\rightarrow \mathrm{fcc} \rightarrow$ dhcp (cf. Fig. 2b). The surface analysis carried out with the interference microscopy methods indicates that different tilts are occurring that cancel each other (within about $1 \mu \mathrm{m}$ ).

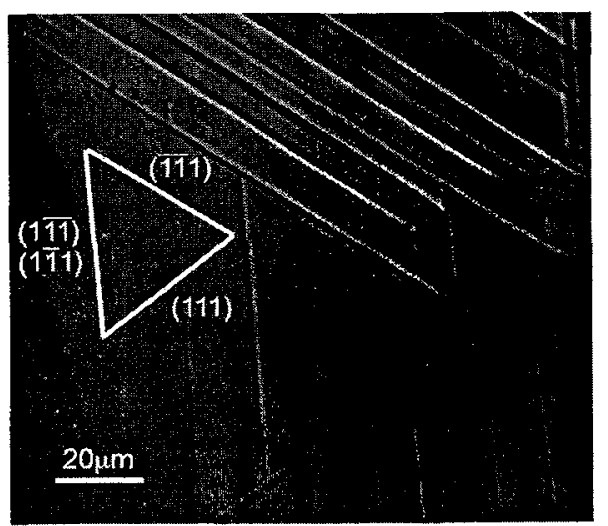

a)

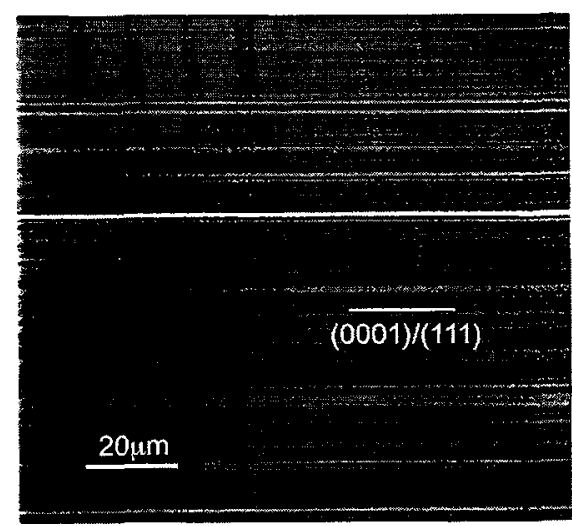

b)

Figure 2: Light optical images (DIC) of transformation induced surface markings. (a) Co- 6.0at.\%Fe (surface normal $\left.[1 \overline{\mathrm{I}} 0]_{\mathrm{fcc}}\right)$. After the transformation $\mathrm{fcc} \rightarrow \mathrm{dhcp}$ fine striations are observed parallel to the traces of the four $\{111\} \mathrm{fcc}$ habit planes. Within each striation different tilts are cancelling each other almost completely. (b) Co- 1.5at.\%Fe (surface normal

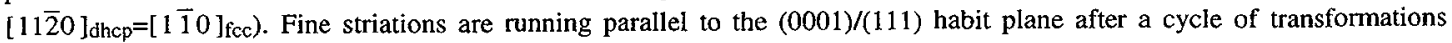
$\mathrm{dhcp} \rightarrow \mathrm{fcc} \rightarrow \mathrm{dhcp}$. Different tilts are cancelling each other within a range of $\sim 1 \mu \mathrm{m}$.

\subsection{Transformation induced dislocations and stacking faults}

After the thermally induced transformation $\mathrm{fcc} \rightarrow \mathrm{dhcp}$ in Co- 1.5 at. \% Fe a high density $\left((2 \pm 1.5) 10^{13} \mathrm{~m}^{-2}\right)$ of grown-in sessile dislocations is observed (cf. Fig. 3a). The dislocations are lying on the close packed planes $(0001)_{\mathrm{dhcp}}$ which are edge on in the TEM projection. Since the dislocations show strong contrast in $g=[0004]_{\mathrm{dhcp}}$ their Burgers vector contains a component normal to the close packed glide planes; 
therefore the dislocations are sessile. As shown by image simulation this component is $\mathbf{c} / 4$ (c= $[0001]_{\mathrm{dhcp}}$ denotes the dhcp lattice vector normal to the close packed planes). Experimental images taken in different $\mathbf{g}$ vectors shows that the Burgers vector contains an additional component $\mathbf{p}$ in the glide plane (p $=1 / 3<1 \overline{1} 00>_{\text {dhcp }}=1 / 6<112>_{\text {fcc }}$ denotes a Shockley partial Burgers vector) leading to a total Burgers vector of $\mathbf{c} / 4+\mathbf{p}$. Frequently, the dislocations are running along a close packed direction $\left(<11 \overline{2} 0>_{\text {dhcp }}\right)$ and occur in a dipolar configuration. Since $\mathbf{c} / 4+\mathbf{p}$ is not a lattice vector of the dhcp structure the dislocations are partial dislocations bounding stacking faults (cf. Fig. 3b). The density of the stacking faults is about $(1.2 \pm 0.2) 10^{7} \mathrm{~m}^{-1}$.

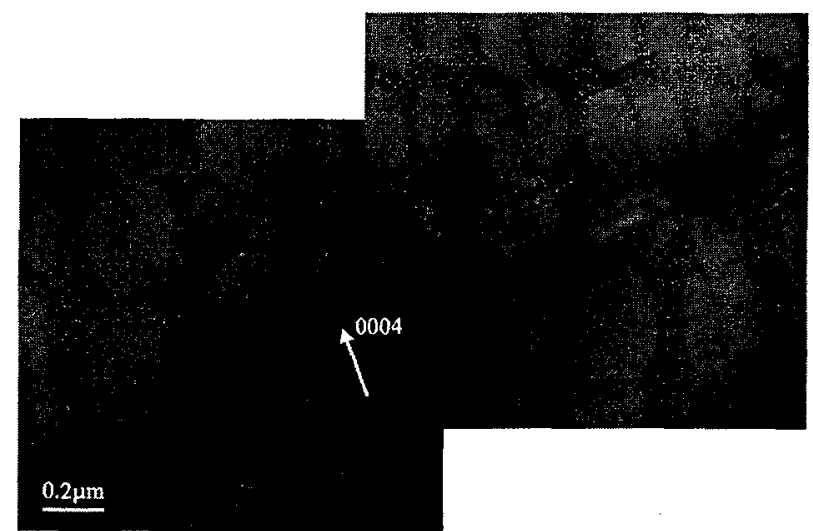

a)

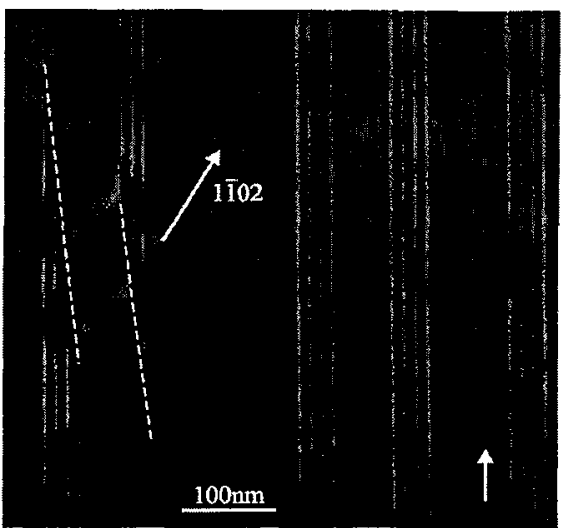

b)

Figure 3: Co- 1.5at.\%Fe; dhcp phase. Transformation induced dislocations and stacking faults. (a) A high density of straight, sessile partial dislocations (Burgers vector $c / 4+p$ ) occurs in the dhcp lattice. The dislocations are lying on the close packed

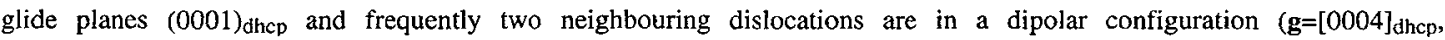
$\left.\mathbf{B D} \approx \mathbf{F N}=[1 \overline{12} 0]_{\mathrm{dhcp}}\right)$. (b) A high density of stacking faults lies on the close packed planes. Stacking faults with $\mathbf{R}=\mathbf{c} / 4+\mathbf{p}$ are terminating at the sessile partial dislocations (a dislocation dipole is marked by two dashed lines). Frequently stacking faults

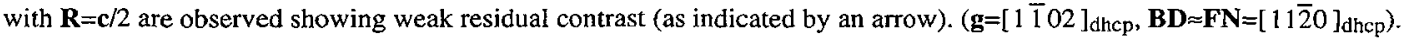

The stacking faults were analysed in detail by HRTEM methods (cf. Fig. 4). The close packed layers are edge on and defocus conditions were set to show bright spots corresponding to the projected atomic columns. Therefore the stacking sequence is revealed directly in the HRTEM image. The stacking faults in Fig. 4a to $c$ (denoted $I_{1}, I_{2}$ and $E$ ) have the fault vector $\mathbf{R}=\mathbf{c} / 4+\mathbf{p}, \mathbf{c} / 2$ and $3 \mathbf{c} / 4+\mathbf{p}$, respectively. In the bright field image of Fig. $3 \mathrm{~b}$ the stacking faults $\mathrm{I}_{1}$ and $\mathrm{E}$ lead to similar strong contrast since the phase factor $\alpha=2 \pi \mathrm{g} \cdot \mathbf{R}=\pi / 3$. Contrary to this, $\alpha=2 \pi$ in the case of $I_{2}$ and no contrast should occur. However, faint residual contrast is still visible indicating a slight supplementary displacement caused by a change in the interatomic spacing near the faults.

\section{DISCUSSION}

\subsection{Disconnections at the transformation front}

According to the structural model outlined in [3] the transformation $\mathrm{fcc} \rightarrow$ dhcp can be achieved when two transformation dislocations on neighbouring layers are occurring on every fourth close packed plane. Since the transformation dislocations can have any one out of three possible Shockley partial Burgers vectors $\mathbf{p}_{\mathrm{i}}\left(\Sigma \mathbf{p}_{\mathrm{i}}=0\right)$ attractive interactions (e.g. according to the relation $\left.\mathbf{p}_{1}+\mathbf{p}_{2}=-\mathbf{p}_{3}\right)$ can occur. In Co6.0at.\%Fe the transformation dislocations on adjacent close packed planes attract each other. They are gliding as a pair and combine to a single transformation dislocation; these paired dislocations have one (e.g. $-\mathbf{p}_{3}$ ) out of the three possible Shockley partial Burgers vectors $-\mathbf{p}_{\mathrm{i}}$ only (cf. Fig. 1). Since at each paired dislocation an interfacial step is occurring that is four close packed layers in height $(h=0.8 \mathrm{~nm})$ the 
tapering transformation fronts are made up by interfacial defects with both step and dislocation character: disconnections $\left(-\mathrm{p}_{3}, \mathrm{~h}=0.8 \mathrm{~nm}\right.$ ) (a general concept of disconnections is outlined in [6]). At the transformation front the disconnections repel each other forming a pile-up. Although the consecutive glide of the disconnections having the same Burgers vector could be explained by a pole mechanism direct experimental evidence of a polar dislocation was not obtained. Finally, it should be noted that the transformation mechanism is similar to that of the transformation $\mathrm{fcc} \rightarrow \mathrm{hcp}$ in $\mathrm{Co}-32 \mathrm{at}$.\% Ni. In this case disconnections ( $\mathbf{p}, \mathrm{h}=0.4 \mathrm{~nm}$ ) having the same Shockley partial Burgers vector (bounding intrinsic stacking faults) were observed to glide on every other close packed plane [7].

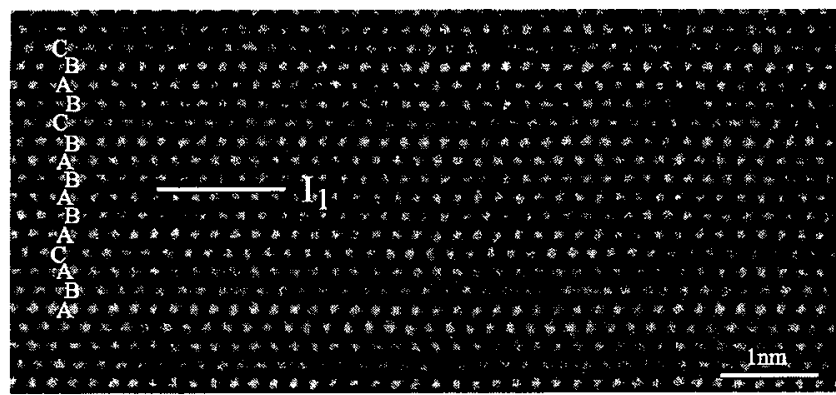

a)

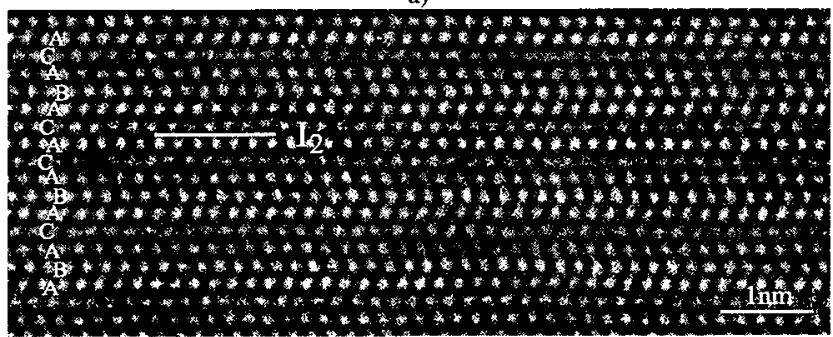

b)

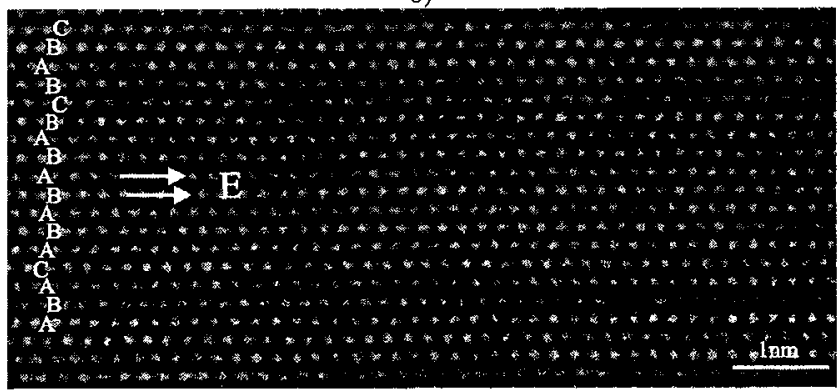

c)

Figure 4: Co- 1.5at.\%Fe; dhcp phase. HRTEM images of stacking faults $\left(\mathbf{B D}=\mathbf{F N}=[11 \overline{2} 0]_{\text {dhcp. }}\right.$. The stacking sequence is revealed directly. (a) Intrinsic fault $I_{1}$ (marked by a line, $R=c / 4+p$ ). (b) Intrinsic fault $I_{2}$ (marked by a line, $R=c / 2$ ). Extrinsic fault $E$ (the two layers not belonging to the dhcp stacking sequence are marked by arrows, $R=3 c / 4+p$ ).

\subsection{Transformation induced strains}

\subsubsection{Surface striations}

Since all the paired transformation dislocations at the front of a dhcp lamella have the same Burgers vector they cause long range strains. The resolved shear strain of $18 \%$ leads to local tilts at free crystal surfaces (cf. Fig. 2). It is concluded from the surface analysis that dhcp lamellae have an average thickness of about $300 \mathrm{~nm}$ (corresponding to an average of about 400 paired transformation dislocations of 
the same $-\mathbf{p}_{\mathrm{i}}$ ). Lamellae having different shear strains (along the three different $-\mathbf{p}_{\mathrm{i}}$ ) occur about equally. Therefore the total shear strain is cancelled at a mesoscopic scale (within about $1 \mu \mathrm{m}$ ) in agreement with the experimental results. Similar results were obtained in the case of the thermally induced transformation $\mathrm{fcc} \rightarrow$ hcp in Co- 32at.\% Ni [7].

\subsubsection{Strain induced dislocations and stacking faults}

The Burgers vectors $\pm(c / 4+p)$ of the sessile dislocations in the dhcp lattice (cf. Fig. 3) correspond to those of total glide dislocations in the fcc parent phase. Therefore, it is concluded that in the fcc matrix slip is activated caused by the transformation shear strains during the thermally induced transformation $\mathrm{fcc} \rightarrow$ dhcp. Subsequently the fcc glide dislocations are incorporated in the dhcp lattice by the moving transformation front leading to the observed structure of grown-in dislocations. Similar conclusions were made in the case of the transformation fcc $\rightarrow$ hcp in pure Co [8] and CoNi [7]; it should be noted that the fcc glide dislocations can react with the transformation dislocations $\mathbf{p}_{\mathrm{i}}$. This can lead to Burgers vectors $\pm(\mathbf{c} / 4-\mathbf{p})$ (e.g. by the reaction $\left.\left(\mathbf{c} / 4+\mathbf{p}_{1}\right)+\mathbf{p}_{2}=\left(\mathbf{c} / 4-\mathbf{p}_{3}\right)\right)$.

The partial dislocations $c / 4+p$ are bounding $I_{1}$ stacking faults dragged out by the moving transformation front. Additional reactions of total fcc glide dislocations at the transformation front can occur with previously formed stacking faults. This can either lead to their annihilation or to a change of the type of the stacking fault (from $I_{1}$ to $I_{2}$ and finally to E; cf. Fig. 4).

\subsection{Comparison with the transformation fec $\rightarrow$ dhep in Co- 5.75at. \% Fe}

In single crystals of $\mathrm{Co}-5.75 \mathrm{at} . \% \mathrm{Fe}$ a different mode of transformation is occurring during the thermally induced transformation $\mathrm{fcc} \rightarrow \mathrm{dhcp}$ leading to a microstructure of the dhcp product phase that is completely different from that observed in the present investigation [3]. In Co- 5.75at.\%Fe the glissile transformation fronts contain paired transformation dislocations that have different and compensating Burgers vectors. The transformation dislocations are correlated on an atomic scale since at the interface a repeated sequence of the three possible Burgers vectors $\left(-\mathbf{p}_{1},-\mathbf{p}_{2},-\mathbf{p}_{3}\right)$ is observed. Since the transformation dislocations compensate their long range strain fields completely no strain induced sessile partial dislocations and stacking faults are observed in the dhep product phase of $\mathrm{Co}-5.75 \mathrm{at} . \% \mathrm{Fe}$.

\section{Acknowledgements}

Financial assistance from the Austrian FWF is acknowledged.

\section{References}

1. J.W. Christian, in Martensite, edited by G.B. Olson and M. Cohen (American Society for Metals, Ohio, 1992), p.103.

2. T. Onozuka, S. Yamaguchi, M. Hirabayashi, T. Wakiyama, J. Phys. Soc. Japan 37, 687 (1974)

3. T. Waitz, H.P. Karnthaler, Phil. Mag. A 73, 365 (1996)

4. J.P. Hirth, J. Lothe, in Theory of Dislocations (Krieger Publishing Company, Florida, 1992)

5. P.B. Hirsch, A. Howie, R.B. Nicholson, D. W. Pashley, M.J. Whelan, in Electron Microscopy of Thin Crystals (Butterworth \& Co, London, 1969)

6. J.P. Hirth, R.C. Pond, Acta Mater. 44, 4749 (1996)

7. T. Waitz, H.P. Karnthaler, Acta Mater. 45, 837 (1997)

8. C. Hitzenberger, H.P. Karnthaler, A. Korner, Acta Metall. 36, 2719 (1988) 